\title{
A novel dual correction extended Kalman filtering algorithm for the state of charge real- time estimation of packing lithium-ion batteries.
}

SHI, H., WANG, S., FERNANDEZ, C., YU, C., FAN, Y. and CAO, W.

(C) 2020The Authors. Published by ESG (www.electrochemsci.org). This article is an open access article distributed under the terms and conditions of the Creative Commons Attribution license

(http://creativecommons.org/licenses/by/4.0/). 


\title{
A Novel Dual Correction Extended Kalman Filtering Algorithm for The State of Charge Real-Time Estimation of Packing Lithium-Ion Batteries
}

\author{
HaoTian Shi ${ }^{1}$, Shunli Wang ${ }^{1, *}$, Carlos Fernandez ${ }^{2}$, Chunmei Yu $^{1}$, Yongcun Fan ${ }^{1}$, Wen Cao ${ }^{1}$ \\ ${ }^{1}$ School of Information Engineering, Southwest University of Science and Technology, Mianyang \\ 621010, China; \\ ${ }^{2}$ School of +Pharmacy and Life Sciences, Robert Gordon University, Aberdeen AB10-7GJ, UK. \\ *E-mail: wangshunli@swust.edu.cn
}

Received: 28 August 2020 / Accepted: 7 October 2020 / Published: 31 October 2020

\begin{abstract}
This paper explores the state estimation method of lithium-ion battery pack through theoretical analysis and experimental research. Combining the advantages of the empirical models of various electrochemical models, a new type of composite electrochemistry-dual circuit polarization (E-DCP) model is proposed to better reflect the dynamic performance of the power lithium-ion battery under the conditions of meeting its safe and reliable energy supply requirements. Using the multi-innovation least squares (MILS) algorithm to identify the parameters in the E-DCP model online, so that it has the characteristics of high data utilization efficiency and high parameter identification accuracy. The battery charge and discharge efficiency function is introduced to dynamically modify the battery capacity, and the dynamic function is used to improve the Kalman gain in the extended Kalman filter (EKF), a new type of based on dynamic function improvement and combined with actual capacity correction (FC$\mathrm{DEKF}$ ) algorithm is applied to the estimation of battery pack operating characteristics, which solves the problem that the traditional EKF algorithm is difficult to estimate errors when the system input change rate is large. The experimental results of urban dynamometer driving schedule (UDDS) and complex charge-discharge cycle test show that the maximum error of terminal voltage does not exceed $0.04 \mathrm{~V}$, the accuracy is $99.05 \%$, and the errors of MILS algorithm combined with FC-DEKF algorithm for SOC estimation are all within $1 \%$. The proposed equivalent circuit modeling method and state estimation correction strategy provide a theoretical basis for the reliable application of high-power lithium-ion battery packs.
\end{abstract}

Keywords: electrochemistry-dual circuit polarization model; parameter identification; multiinnovation least squares; improved extended Kalman filter; dynamic function optimization; lithium-ion batteries 


\section{INTRODUCTION}

With the consumption of fossil energy and the pressure of environmental protection, as well as the limitation of the maximum capacity of battery cell, it has become more and more important to improve the mathematical modeling research of battery group management technology [1-4]. A largecapacity battery system generally consists of multiple battery cell connected in parallel and converged, and then encapsulated by battery cells shell and cell covers to form multiple battery cells cascaded. It has the characteristics of large capacity and many series and parallel nodes [5-8]. For the entire battery pack system, its stability and real-time and effective status monitoring are the prerequisites for the system's vitality $[9,10]$. The development of power battery pack applications has entered a new era. Accurate battery models and accurate state estimation are of great significance for predicting the range of battery packs.

Lithium-ion battery is a typical nonlinear system. Its work involves multiple processes such as electrochemical reaction and energy conversion. The traditional pure electrochemistry or pure equivalent circuit model is not enough to fully reflect its multiple mutual coupling processes. Carry out composite modeling optimization of lithium-ion battery, and use different working conditions to test, and experiment with the accurate expression of lithium-ion battery model [11-15]. Aiming at difficult problems in the safety management of power battery packs under the influence of complex working conditions, single combination structure and environmental conditions, the equivalent model is constructed and researched, based on the exploration of working characteristics under different working conditions, combined with the characteristic information of external measurable parameters, and the establishment is related to time The equivalent model of, and the description of the state space, lay the foundation for the subsequent accurate estimation of the battery state [16-18].

As the most widely used estimation method in recent years, the accuracy of State of Charge (SOC) estimation has been constantly improved [19, 20]. Accurate SOC estimation helps to improve the safety level and cycle life performance of the energy power supply system. Based on achieving this goal, while constructing an accurate composite electrochemical circuit model, it is combined with a highprecision SOC filter to achieve real-time monitoring of SOC [21, 22]. Wang et al. used the Unscented Particle Filter to predict the state of electric charges [23]. In the literature [24], an equivalent neural network circuit model was established and SOC estimation was performed based on this model.

Research the implementation technology of the online accurate estimation management system of the power battery pack, and ensure the safety and reliability of the battery pack application through key technology research such as the detection of key parameters of the battery pack and its internal monomers [25, 26].In the literature [27], an SOC estimation algorithm is applied to the battery with degradation tracking. A method of SOC estimation based on a white box equivalent neural network circuit model was proposed [28]. Luo et al. studied a method for estimating SOC of lithium-ion batteries based on the Cubature Kalman filter [29]. Guo et al. used the cyclic neural network and the genetic algorithm to estimate SOC of lithium-ion batteries [30]. Hou et al. used an adaptive double unscented Kalman filter (UKF) for normal-Gamma estimation of battery parameters and charged state [31].

Most of the existing studies have not considered the influence of the system input change rate on the accuracy of the state estimation of the group of batteries. By analyzing the advantages and 
disadvantages of electrochemical and equivalent circuit models, a new type of composite electrochemistry-dual circuit polarization (E-DCP) model is proposed under the conditions of meeting its safe and reliable energy supply requirements, which better reflects the power lithium-ion battery's step-by-step process. Through theoretical analysis and experimental research, combined with the characteristic information of external measurable parameters, establish a time-related equivalent model and describe the state space. The battery charge and discharge efficiency function is introduced to dynamically modify the battery capacity, and the dynamic function is used to improve the Kalman gain in the extended Kalman filter (EKF), which realizes the accurate estimation of the state of the group battery when the system input change rate is high. Through urban dynamometer driving schedule (UDDS) and complex charge-discharge cycle test experiments, the accuracy of the E-DCP model and the accuracy of the multi-innovation least squares (MILS) algorithm combined with the EKF algorithm based on dynamic function improvement and combined with actual capacity correction (FC-DEKF) algorithm for SOC estimation are verified. This kind of modeling and collaborative prediction correction strategy research is of great significance to improve the estimation accuracy and robustness of highpower battery packs.

\section{THEORETICAL ANALYSIS}

\subsection{Electrical equivalent modeling}

The quality of the lithium-ion battery model directly affects the accuracy of the subsequent SOC estimation. In order to realize the accurate modeling of the lithium-ion battery, this paper explores the output characteristics of the closed-circuit voltage during the working process of the lithium-ion battery pack through mechanism analysis and simulation experiments of working conditions.
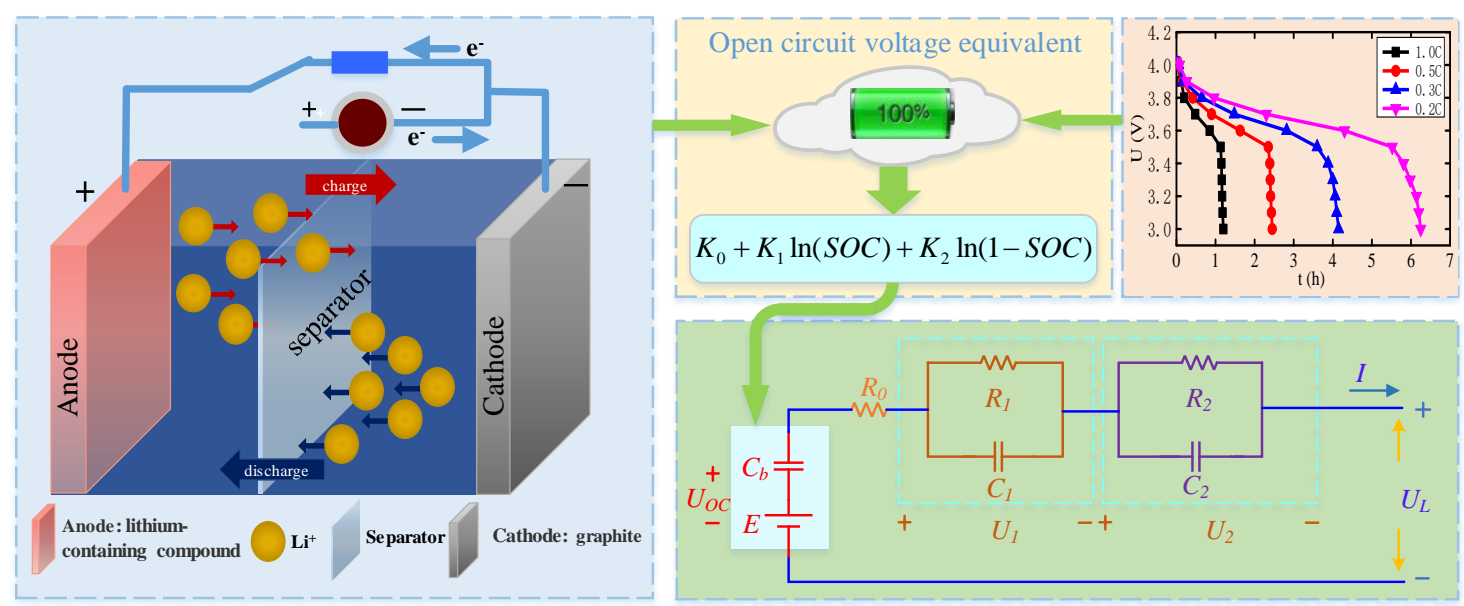

Figure 1. Electrical equivalent modeling 
In order to more accurately reflect the changing law of the battery, using the optimized electrochemical Nernst model and combining the second-order circuit equivalence, a new type of complex electrochemistry-dual circuit polarization (E-DCP) model is proposed to realize the working process precise description. The equivalent structure of each part in the compound electrochemical-dual circuit polarization model is shown in Fig. 1.

In Fig. $1, R_{0}$ is the battery ohmic resistance, which is the instantaneous voltage drop caused by battery current; $R_{1}$ and $R_{2}$ the polarization resistance of the battery; $C_{1}$ and $C_{2}$ are the polarization capacitance of the battery. The parallel circuit composed of $R_{1}$ and $C_{1}$ has a large time constant; the parallel circuit composed of $R_{2}$ and $C_{2}$ has a small time constant; $I$ is the loop current of the battery; $U_{L}$ is the battery terminal voltage. $E$ is the ideal voltage source. $E$ is used together with $C_{b}$ as a whole to represent the change of open circuit voltage $U o c$. The effect of charge-discharge rate gradient change on energy attenuation law is analyzed and summarized. The E-DCP model combines the advantages of both the electrochemical model and the equivalent circuit model, studies the effect of the charge-discharge rate on the internal battery chemical reaction change, and uses the double RC circuit to specify the polarization response of the battery to better present the dynamic performance of the power lithium-ion battery under step working conditions.

Through the study of E-DCP model, based on the exploration of working characteristics under different working conditions, combined with the characteristic information of external measurable parameters, the time-related equivalent model is established and the state space description is shown in Equation (1).

$$
\left\{\begin{array}{l}
d U_{1} / d t=I_{t} / C_{1}-U_{1} / R_{1} C_{1} \\
d U_{2} / d t=I_{t} / C_{2}-U_{2} / R_{2} C_{2} \\
U_{L}=U_{O C}\left(S O C_{t}\right)-I_{t} R_{0}-U_{1}-U_{2}
\end{array}\right.
$$

where $t$ is the continuous time constant, $I_{t}$ and $S O C_{t}$ represent the current and SOC values in the continuous time state.

The first-order backward difference principle is used to discretize equation 1, and the results are shown in Equation (2).

$$
\left\{\begin{array}{l}
{\left[U_{1}(k)-U_{1}(k-1)\right] / T=-U_{1}(k) / R_{1} C_{1}+I(k) / C_{1}} \\
{\left[U_{1}(k-1)-U_{1}(k-2)\right] / T=-U_{1}(k-1) / R_{1} C_{1}+I(k-1) / C_{1}} \\
{\left[U_{2}(k)-U_{2}(k-1)\right] / T=-U_{2}(k) / R_{2} C_{2}+I(k) / C_{2}} \\
{\left[U_{2}(k-1)-U_{2}(k-2)\right] / T=-U_{2}(k-1) / R_{2} C_{2}+I(k-1) / C_{2}}
\end{array}\right.
$$

where $k$ is a discrete time constant, and $T$ is the sampling time.

By combining equations 1 and 2, the system difference equation suitable for full parameter identification is obtained as shown in Equation (3).

$$
U_{L}(k)=c_{0}+c_{1} U_{L}(k-1)+c_{2} U_{L}(k-2)+c_{3} I(k)+c_{4} I(k-1)+c_{5} I(k-2)
$$

where $c_{0}$ to $c_{5}$ are the coefficients of the differential equation. The E-DCP model combines the advantages of both the electrochemical model and the equivalent circuit model, and uses the double resistance-capacitance (RC) circuit to specify the polarization response of the battery, which laid a foundation for the subsequent parameter identification and accurate estimation of the battery state. 


\subsection{Full-parameter online identification}

To improve data utilization, the MILS algorithm is used for online parameter identification for the lithium-ion battery. This algorithm extends the innovation into vector innovation or matrix innovation, and can more fully use the scalar innovation of each moment in the iteration calculation process to improve data usage efficiency, accelerate algorithm convergence and increase parameter identification accuracy. The full-parameter identification process of using the MILS algorithm to estimate the parameters in the E-DCP model, as shown in Fig. 2.

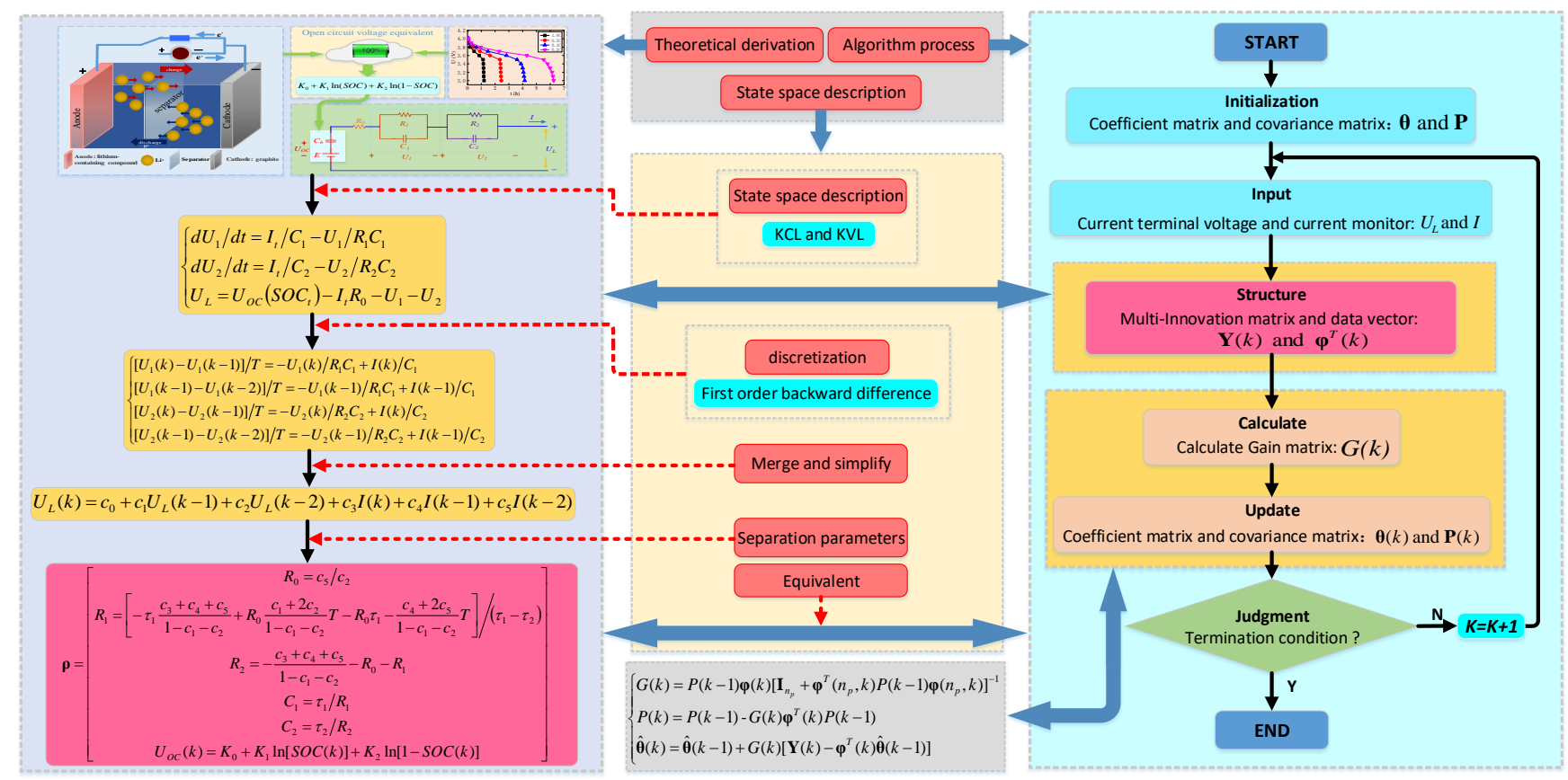

Figure 2. Equation of State discretization and iterative Flow chart with MILS algorithm.

The MILS algorithm performs recursion for solution innovation in each time step, and the calculation quantity is not high. In order to simplify the state space representation of the model, equation (3) is converted to the least squares form:

$$
y(k)=\varphi^{T}(k) \boldsymbol{\theta}
$$

where $y(k)$ said the system output vector, $\boldsymbol{\varphi}(k)$ and $\boldsymbol{\theta}$ represent system data and parameter vector respectively. The $y(k), \boldsymbol{\varphi}(k)$ and $\boldsymbol{\theta}$ specific said is as follows:

$$
\left\{\begin{array}{l}
y(k)=U_{L}(k) \\
\boldsymbol{\varphi}(k)=[1, U(k-1), U(k-2), I(k), I(k-1), I(k-2)]^{T} \\
\boldsymbol{\theta}=\left[c_{0}, c_{1}, c_{2}, c_{3}, c_{4}, c_{5}\right]^{T} \\
\boldsymbol{\rho}=\left[R_{0}, R_{1}, R_{2}, C_{1}, C_{2}, U_{O C}\right]^{T}
\end{array}\right.
$$

where $\rho$ is the parameter matrix to be identified in the E-DCP model. With the simultaneous equations of (1) and (2), the calculation results of $\boldsymbol{\theta}$ can be obtained: 


$$
\left\{\begin{array}{l}
\left.c_{0}=\left(1-c_{1}-c_{2}\right) U_{o C}(k), c_{1}=2 \tau_{1} \tau_{2}+\left(\tau_{1}+\tau_{2}\right) T\right] /\left[\tau_{1} \tau_{2}+\left(\tau_{1}+\tau_{2}\right) T+T^{2}\right. \\
c_{2}=-\tau_{1} \tau_{2} /\left[\tau_{1} \tau_{2}+\left(\tau_{1}+\tau_{2}\right) T+T^{2}\right], c_{5}=-R_{0} \tau_{1} \tau_{2} /\left[\tau_{1} \tau_{2}+\left(\tau_{1}+\tau_{2}\right) T+T^{2}\right] \\
c_{3}=-\left[R_{0} \tau_{1} \tau_{2}+R_{0}\left(\tau_{1}+\tau_{2}\right) T+R_{1} \tau_{2} T+R_{2} \tau_{1} T+\left(R_{0}+R_{1}+R_{2}\right) T^{2}\right] /\left[\tau_{1} \tau_{2}+\left(\tau_{1}+\tau_{2}\right) T+T^{2}\right] \\
c_{4}=\left[2 R_{0} \tau_{1} \tau_{2}+R_{0}\left(\tau_{1}+\tau_{2}\right) T+R_{1} \tau_{2} T+R_{2} \tau_{1} T\right] /\left[\tau_{1} \tau_{2}+\left(\tau_{1}+\tau_{2}\right) T+T^{2}\right]
\end{array}\right.
$$

where the calculation of $\tau_{1}$ and $\tau_{2}$ is shown in Equation (7).

$$
\left\{\begin{array}{l}
\tau_{1}=\max \left(\frac { 1 } { 2 } \left[\frac{c_{1}+2 c_{2}}{1-c_{1}-c_{2}}+\sqrt{\left.\left.\left(\frac{c_{1}+2 c_{2}}{1-c_{1}-c_{2}}\right)^{2}+\frac{4 c_{2} T^{2}}{1-c_{1}-c_{2}}\right], \frac{1}{2}\left[\frac{c_{1}+2 c_{2}}{1-c_{1}-c_{2}}-\sqrt{\left(\frac{c_{1}+2 c_{2}}{1-c_{1}-c_{2}}\right)^{2}+\frac{4 c_{2} T^{2}}{1-c_{1}-c_{2}}}\right]\right)}\right.\right. \\
\tau_{2}=\min \left(\frac{1}{2}\left[\frac{c_{1}+2 c_{2}}{1-c_{1}-c_{2}}+\sqrt{\left(\frac{c_{1}+2 c_{2}}{1-c_{1}-c_{2}}\right)^{2}+\frac{4 c_{2} T^{2}}{1-c_{1}-c_{2}}}\right], \frac{1}{2}\left[\frac{c_{1}+2 c_{2}}{1-c_{1}-c_{2}}-\sqrt{\left(\frac{c_{1}+2 c_{2}}{1-c_{1}-c_{2}}\right)^{2}+\frac{4 c_{2} T^{2}}{1-c_{1}-c_{2}}}\right]\right)
\end{array}\right.
$$

By further separating equation (6), the calculation results of each parameter in the E-DCP model are shown in Equation (8).

$$
\left\{\begin{array}{l}
R_{0}=c_{5} / c_{2}, R_{2}=-\frac{c_{3}+c_{4}+c_{5}}{1-c_{1}-c_{2}}-R_{0}-R_{1}, C_{1}=\tau_{1} / R_{1}, C_{2}=\tau_{2} / R_{2} \\
R_{1}=\left[-\tau_{1} \frac{c_{3}+c_{4}+c_{5}}{1-c_{1}-c_{2}}+R_{0} \frac{c_{1}+2 c_{2}}{1-c_{1}-c_{2}} T-R_{0} \tau_{1}-\frac{c_{4}+2 c_{5}}{1-c_{1}-c_{2}} T\right] /\left(\tau_{1}-\tau_{2}\right)
\end{array}\right.
$$

High usage efficiency of innovation can be achieved by using the MILS algorithm for fullparameter online identification in the E-DCP model and combining the parameter separation result after system discretization. The output matrix of MILS algorithm is shown in Equation (9).

$$
\mathbf{Y}(k)=\boldsymbol{\varphi}^{T}(k) \boldsymbol{\theta}(k-1)+(p, k) \Leftarrow \mathbf{V}(p, k)=\left[\begin{array}{c}
y(k)-\boldsymbol{\varphi}^{T}(k) \hat{\boldsymbol{\theta}}(k-1) \\
y(k-1)-\boldsymbol{\varphi}^{T}(k-1) \hat{\boldsymbol{\theta}}(k-2) \\
\vdots \\
y(k-p+1)-\boldsymbol{\varphi}^{T}(k-p+1) \hat{\boldsymbol{\theta}}(k-p)
\end{array}\right]
$$

where $\mathbf{Y}(\mathrm{k})$ is the output matrix of the algorithm, $p$ is the length of the matrix innovation, and $\hat{\boldsymbol{\theta}}(k-1)$ is the estimated value of the parameter vector at the previous moment.

By using equation (5-8) to calculate the parameters at each moment in the E-DCP model, combined with the output matrix of MILS algorithm, the parameter values were iteratively updated through Equation (10).

$$
\left\{\begin{array}{l}
G(k)=P(k-1) \boldsymbol{\varphi}(k)\left[\mathbf{I}_{n_{p}}+\boldsymbol{\varphi}^{T}\left(n_{p}, k\right) P(k-1) \boldsymbol{\varphi}\left(n_{p}, k\right)\right]^{-1} \\
P(k)=P(k-1)-G(k) \boldsymbol{\varphi}^{T}(k) P(k-1) \\
\hat{\boldsymbol{\theta}}(k)=\hat{\boldsymbol{\theta}}(k-1)+G(k)\left[\mathbf{Y}(k)-\boldsymbol{\varphi}^{T}(k) \hat{\boldsymbol{\theta}}(k-1)\right]
\end{array}\right.
$$

Where $P(k)$ is the covariance matrix of MILS algorithm, $G(k)$ is the correction factor in the MILS algorithm, which ensures a relative small terminal voltage error and a relative fast convergence speed in each iteration of the algorithm. Through the above theoretical analysis, the online accurate identification of full-parameter based on E-DCP model is realized.

\subsection{Iterative calculation}

The Kalman filter algorithm gives high optimization estimation accuracy in a linear system, but as battery SOC estimation is a typical non-linear system, it is difficult to use the traditional Kalman filter 
algorithm in the battery management system (BMS). The EKF algorithm is one of the most common SOC estimation algorithms. This algorithm not only solves the state estimation problem in a non-linear system but also ensures fast correction and therefore achieves adaptive target tracking in the event of a relative big error of the initial value [32]. In this paper, MILS and EKF are combined to improve the robustness of BMS and the accuracy of SOC, so as to achieve accurate estimation of Lithium-ion battery status. The algorithm iteration process is shown in Fig. 3.

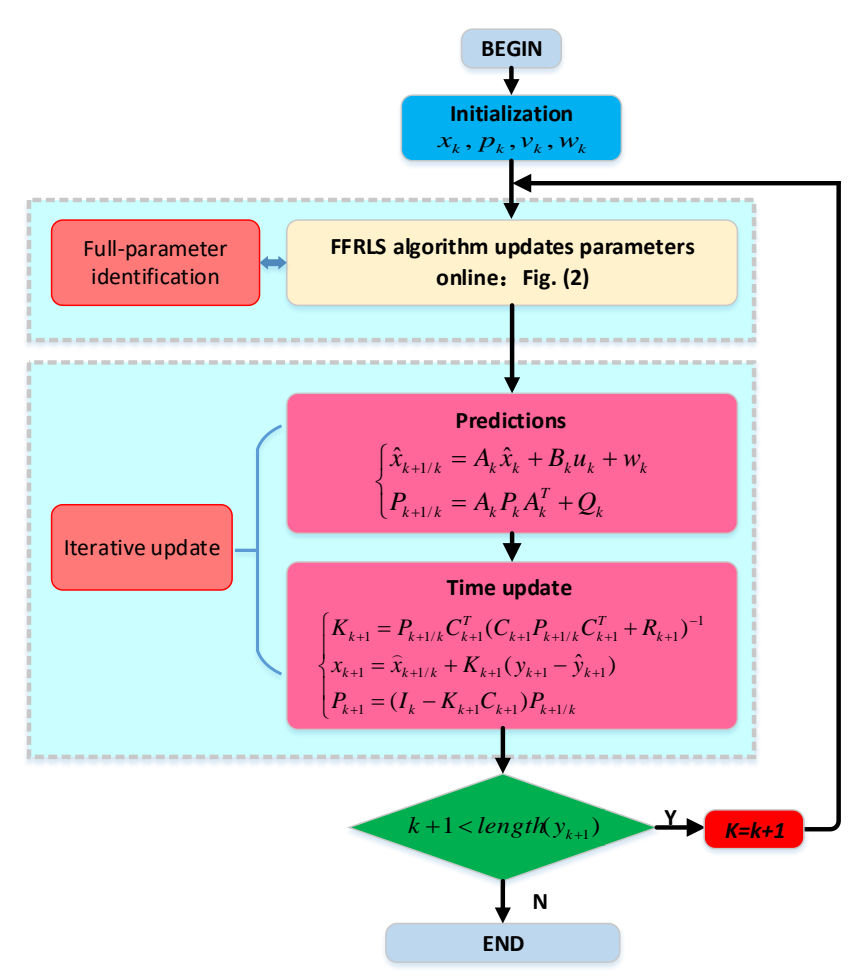

Figure 3. The EKF algorithm iteration flowchart

To use the EKF algorithm, with $S O C, U_{1}$ and $U_{2}$ in the state equation as the state variables, current $I$ as the system input and $U o c$ as the system output, the expression of the state equation and the observation equation is constructed, as shown in Equation (11).

$$
\left\{\begin{array}{l}
x_{k}=A_{k-1} x_{k-1}+B_{k-1} x_{k-1}+w_{k-1} \\
y_{k}=C_{k} x_{k}+D_{k-1} u_{k}+v_{k}
\end{array}\right.
$$

where $x$ is the system state variable, $u$ is the system input and $y$ is the system output, $w_{k}$ and $v_{k}$ are the system noise, with the covariance matrices being $Q$ and $R$ respectively. The $\mathbf{A}, \mathbf{B}, \mathbf{C}$ and $\mathbf{D}$ matrix of the above-mentioned formula is expressed as follows:

$$
\left\{\begin{array}{l}
A_{k}=\left[\begin{array}{ccc}
1 & 0 & 0 \\
0 & \exp \left(-T / \tau_{1}\right) & 0 \\
0 & 0 & \exp \left(-T / \tau_{2}\right)
\end{array}\right], C_{k}=\left[\frac{d U_{O C}(S O C)}{d(S O C)}, 1,1\right]^{T} \\
B_{k}=\left[-\eta T / Q_{N}, R_{1}\left[1-\exp \left(-T / \tau_{1}\right)\right], R_{2}\left[1-\exp \left(-T / \tau_{2}\right)\right]\right], D_{k}=R_{0}
\end{array}\right.
$$


The EKF algorithm uses the first-order Taylor expansion of the open-circuit voltage equation to transform the nonlinear system into a linear system, and its calculation is shown in Equation (13).

$$
\frac{d U_{O C}(S O C)}{d(S O C)}=\frac{K_{1}}{S O C}-\frac{K_{2}}{1-S O C}
$$

The parameter values estimated in real time by MILS algorithm and EKF algorithm are combined to realize the iterative calculation of SOC values. The calculation of state prediction equation and state covariance prediction equation is shown in Equation (14).

$$
\left\{\begin{array}{l}
\hat{x}_{k+1 / k}=A_{k} \hat{x}_{k}+B_{k} u_{k}+w_{k} \\
P_{k+1 / k}=A_{k} P_{k} A_{k}^{T}+Q_{k}
\end{array}\right.
$$

where $P_{k}$ is the covariance matrix.

In order to obtain the optimal filter gain matrix $K_{k+1}$, the optimal state matrix $x_{k+1}$ and the optimal covariance matrix value $P_{k+1}$, the above prediction equation needs to be updated over time, and the update equation is shown in Equation (15).

$$
\left\{\begin{array}{l}
K_{k+1}=P_{k+1 / k} C_{k+1}^{T}\left(C_{k+1} P_{k+1 / k} C_{k+1}^{T}+R_{k+1}\right)^{-1} \\
x_{k+1}=\widehat{x}_{k+1 / k}+K_{k+1}\left(y_{k+1}-\hat{y}_{k+1}\right) \\
P_{k+1}=\left(I_{k}-K_{k+1} C_{k+1}\right) P_{k+1 / k}
\end{array}\right.
$$

By combining with the above-mentioned EKF optimal iteration process, the MILS algorithm is used to identify the parameters of the E-DCP model, to achieve state estimation of the BMS and improve SOC estimation precision.

\subsection{Adaptive capacity correction}

Throughout the life cycle of a lithium-ion battery pack, due to the decomposition of the electrolyte inside the battery and the accumulation of the passivation layer of the solid electrolyte interface film on the surface of the positive electrode, the charge and discharge efficiency will change with electrochemical degradation [33]. In order to solve the above problems, this paper introduces the battery charging and discharging efficiency function to dynamically modify the battery capacity, so that in practical applications, the rated capacity of the lithium-ion battery pack more accurately reflects the actual capacity, and the adaptive correction of the battery capacity is always realized. The function is shown in Equation (16).

$$
\left\{\begin{array}{l}
Q_{C}=f\left(\varepsilon_{C}\right) Q_{N} \\
Q_{D}=f\left(\varepsilon_{D}\right) Q_{N}
\end{array}\right.
$$

where $Q_{N}$ is the rated capacity of the lithium-ion battery pack, $Q_{C}$ and $Q_{D}$ are the actual battery capacity corrected by the charge and discharge efficiency function, $f\left(\varepsilon_{C}\right)$ and $f\left(\varepsilon_{D}\right)$ are the charge efficiency correction function and the discharge efficiency correction function, respectively, and are the charge rate and discharge rate, respectively.

In order to obtain the charge-discharge efficiency correction function, it is necessary to obtain the coulomb efficiency under different charge-discharge rates. The calculation is shown in Equation (17). 


$$
\left\{\begin{array}{l}
\eta_{C}=Q_{C n} / Q_{C n, \text { max }}, n=n_{1} C, n_{2} C, \ldots, n_{5} C \\
\eta_{D}=Q_{D m} / Q_{D m, \text { max }}, m=m_{1} C, m_{2} C, \ldots, m_{5} C
\end{array}\right.
$$

where $\eta_{C}$ and $\eta_{D}$ are the efficiency of charging and the efficiency of discharging respectively, $Q_{C n}$ is the charge capacity when the charge rate is $n$, and $Q_{D m}$ is the discharge capacity when the discharge rate is $m . Q_{C n, \max }$ and $Q_{D n, \max }$ are the maximum charge capacity and maximum discharge capacity, respectively. In this paper, five groups of different current rates are selected for experiments to obtain the corresponding coulomb efficiency, and five groups of different coulomb efficiency data are used as the value range to fit the corresponding values and, so as to realize the adaptive correction of battery capacity.

\subsection{Dynamic function improvement}

The actual application results of a large number of working conditions show that the estimation effect of the EFF algorithm is not ideal when the system input changes rapidly or the instantaneous current change rate is large, and there is even the possibility of divergence. After in-depth analysis and research, it is found that the above situation is caused by the insufficient adjustment of the Kalman gain when the system input changes rapidly or the current instantaneous change rate is large. Based on the above problems, this paper improves the Kalman gain in EKF, uses the idea of one-sided limit, introduces a dynamic function to modify the Kalman gain, and proposes an EKF algorithm based on dynamic function improvement and combined with actual capacity correction (FC-DEKF), which makes EKF suitable for the situation where the system input changes rapidly or the current instantaneous change rate is large. The principle of using the dynamic function to improve the Kalman gain is as follows.

First define the left limit of the rate of change of the system input in the discrete state:

$$
\mathbf{F}_{I-}^{\prime}(k)=\lim _{\Delta t \rightarrow T^{-}} \frac{\Delta I}{\Delta t}=\lim _{\Delta t \rightarrow T} \frac{\mathbf{F}_{I}(k+\Delta t)-\mathbf{F}_{I}(k)}{\Delta t}=\lim _{\Delta t \rightarrow T} \frac{I(k)-I(k-1)}{\Delta t}
$$

where $\mathbf{F}_{I-}^{\prime}(k)$ is the rate of change of the input current of the system at adjacent moments in the discrete state, $\mathbf{F}_{I}(k)$ is the system input at the current moment. Since the actual operation of the system is in a discrete state, its value can be obtained by looking up the table.

Secondly, according to the actual working condition test, the conditions for triggering the dynamic function correction are given:

$$
\mathbf{F}_{I-}^{\prime}(k) \geq \mathbf{F}_{I-}^{\prime}(k)_{\max }
$$

where $\mathbf{F}_{I-}^{\prime}(k)_{\max }$ is the maximum input current change rate that the system can withstand, and its value is obtained according to actual working conditions.

Finally, use the curve characteristics of the exponential function to give the optimized Kalman gain:

$$
K_{k+1}^{\prime}=\phi K_{k+1} \Leftarrow \phi=\left(\gamma+\alpha^{\frac{1}{I_{k}-I_{k-1}}}\right)
$$

where $\phi$ is a dynamic function, $\alpha$ is an empirical factor, and its value is $\alpha \subset(0,1)$. In order to avoid the appearance of "soft adjustment" or "over adjustment" of the dynamic function, a constant factor 
$\gamma$ is introduced to restrict the dynamic function. The value range of the constant factor and the dynamic function are specifically analyzed in the following experimental analysis.

\section{EXPERIMENT ANALYSIS}

\subsection{Experimental test platform}

In order to verify the accuracy of the improved algorithm, this paper uses 7 battery cells connected in series as a sample to conduct an experimental study. The single cells in the sample are connected in parallel and converged by multiple battery cells. During the use of the Lithium-ion battery pack, each battery cell is cascaded together in series. The basic parameters of the experimental sample of the Lithium-ion battery pack are shown in Table 1.

Table 1. The basic performance parameters of the Lib pack samples.

\begin{tabular}{cccc}
\hline No. & Parameter Name & Parameter Value & Brief description \\
\hline 1 & Battery model & $7-\mathrm{ICP}$ & 7 battery cells connected in series \\
2 & Rated capacity & $4.00 \mathrm{Ah}$ & Nominal capacity value \\
3 & Group nominal voltage & $25.90 \mathrm{~V}$ & $\begin{array}{c}\text { Group output rated voltage } \\
\text { Rated voltage of lithium-ion battery } \\
\text { pack }\end{array}$ \\
4 & Cell nominal voltage & $3.70 \mathrm{~V}$ & $\begin{array}{c}\text { Any cell voltage reaches to stop } \\
\text { discharging }\end{array}$ \\
5 & Discharge cut-off voltage & $3.00 \mathrm{~V}$ & Any cell voltage reaches to stop \\
& charging
\end{tabular}

In Table 1 , the $\mathrm{C}$ represents the rated capacity of the battery. According to the selected experimental samples of Lithium-ion battery packs, a small test platform is built using lithium battery test systems, computers, incubators and other equipment to collect and analyze experimental data for Lithium-ion. The test platform can perform real-time data collection on lithium-ion batteries with an upload computer system. The platform structure is shown in Fig. 4. All experiments in this paper are carried out at $25^{\circ} \mathrm{C}$, and the model parameters and algorithm verification at high and low temperatures need to be further improved by subsequent research. 


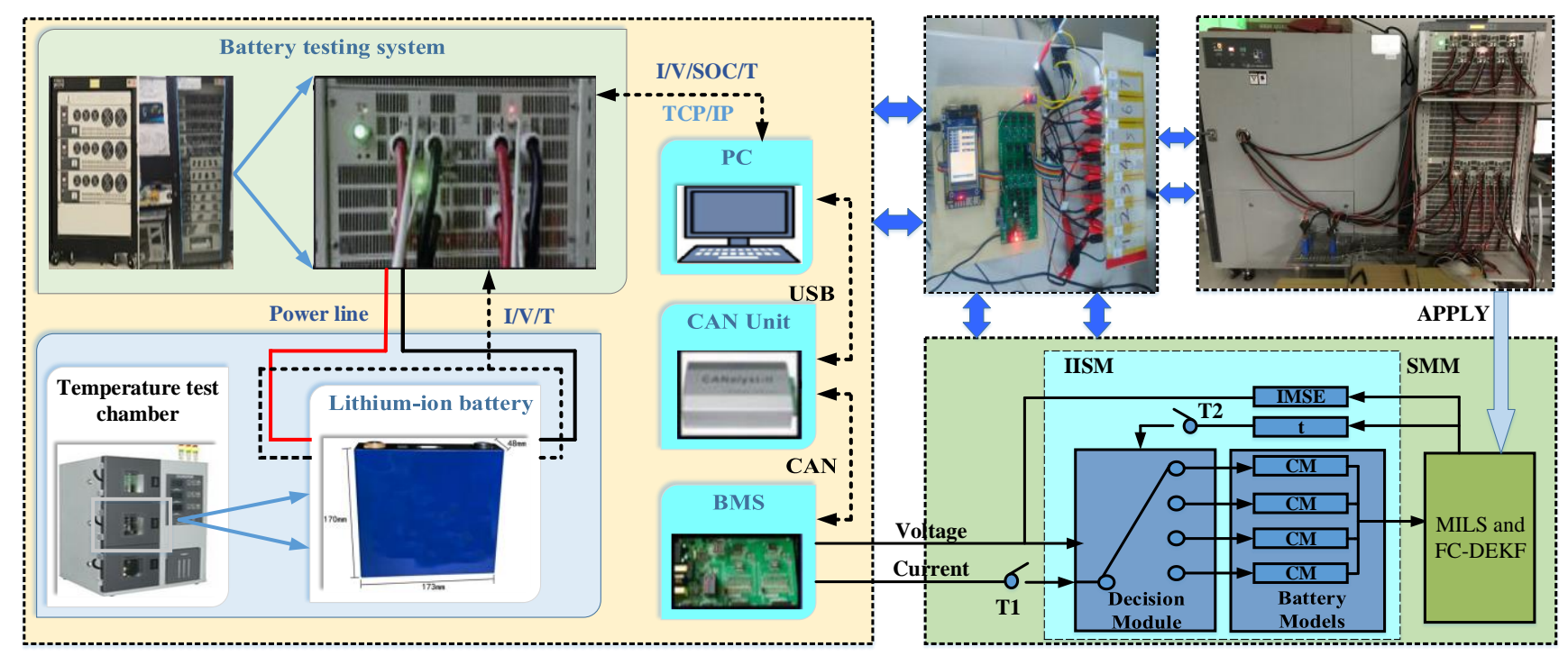

Figure 4. Experimental Li-ion battery test platform

\subsection{Open circuit voltage identification}

Based on the internal redox mechanism of lithium-ion batteries, through the integration and optimization of commonly used electrochemical models, a new type of composite electrochemical-dual circuit polarization model is proposed. The relationship between open circuit voltage (OCV) and SOC of this model is as follows The method of charging and discharging experiment is as follows: (1) Select the experimental sample of the Lithium-ion battery pack and proceed with the discharge current rate of $1 \mathrm{C}$ until the discharge cut-off voltage; (2) Let the experimental sample of the Lithium-ion battery pack stand for 1 hour to make its internal reaction Return to a stable state; (3) Charge the lithium-ion battery pack with constant current at a charging current rate of $0.2 \mathrm{C}$ until the charge cut-off voltage, and then perform constant voltage supplementary charging until the current drops to the charge cut-off current; (4) For lithium The ion battery pack is allowed to stand for 1 hour to restore its internal chemical reaction to a stable state; (5) Discharge the Lithium-ion battery pack at a constant current for half an hour at a discharge current rate of $0.1 \mathrm{C}$ to reduce its SOC to 0.95 ; (6) Allow the sample to stand for 1 hour to return to a stable state, and then record its OCV value; (7) The experiment skips to the fifth step, and the cycle operates 20 times; (8) The aviation lithium-ion battery pack is charged with constant current and voltage until Meet the charging cut-off condition and make the battery fully charged.

Based on the above experimental method, the data set is fitted with the optimized open circuit voltage equation in the E-DCP model, and the OCV-SOC function fitting curve of the above experimental sample is obtained as shown in Figure 5. 


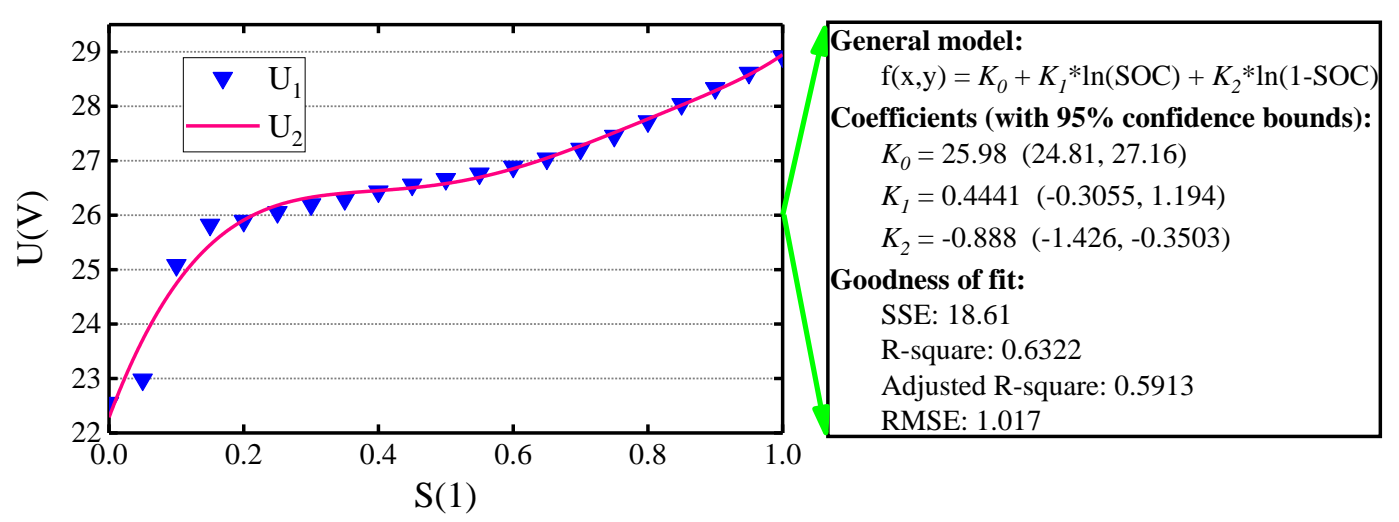

Figure 5. OCV-SOC function fitting curve

In Fig. 5, $\mathrm{U}_{1}$ is the experimental value of open circuit voltage, $\mathrm{U}_{2}$ is the fitting value of open circuit voltage. The $\mathrm{S}$ represents the SOC value, whose unit 1 is the result after normalization. According to the fitting curve shown in Fig. 5, the OCV-SOC change relationship of the test sample can be obtained as shown in Equation (21).

$$
U_{O C}(k)=25.9846+0.4441 * \ln [S O C(k)]-0.8883 * \ln [1-S O C(k)]
$$

It can be seen from Figure 4 that the related coefficient of the fitting curve is 0.6322 , which indicates that the curve fitting accuracy is high and can be used for subsequent parameter identification and precise SOC estimation.

\subsection{Actual capacity correction experiment}

In order to accurately reflect the actual power of the lithium-ion battery pack, five sets of experiments with different current rates are designed to obtain the coulomb efficiency under different charge and discharge rates. The experimental parameters of different charge and discharge current rates are shown in Table 2.

Table 2. Coulomb efficiency under different charge and discharge ratio

\begin{tabular}{cccccc}
\hline Parameter name & First group & Second Group & Third group & Fourth group & Fifth group \\
\hline Charging rate(C) & 0.20 & 0.30 & 0.50 & 1.00 & 1.20 \\
Charging power(Ah) & 3.7599 & 3.7217 & 3.5861 & 3.3844 & 3.2307 \\
$\eta_{C}(1)$ & 1.0000 & 0.9894 & 0.9548 & 0.9001 & 0.8590 \\
\hline \hline Parameter name & First group & Second Group & Third group & Fourth group & Fifth group \\
\hline Discharge rate $(\mathrm{C})$ & 0.10 & 0.20 & 0.50 & 1.00 & 1.20 \\
Discharge power(Ah) & 3.4063 & 3.3941 & 3.3716 & 3.3560 & 3.3500 \\
$\eta_{D}(1)$ & 1.0000 & 0.9964 & 0.9898 & 0.9852 & 0.9835 \\
\hline
\end{tabular}

In Table $2, \eta_{C}$ and $\eta_{D}$ are charging efficiency and discharging efficiency respectively, and the normalized unit is 1 . The units of charge ratio and discharge ratio are $\mathrm{C}$, and $1 \mathrm{C}$ represents the rated capacity of the battery. From the analysis of the experimental results, it can be seen that the charging 
efficiency is the highest under the $0.20 \mathrm{C}$ charge rate condition, and the discharge efficiency under the $0.10 \mathrm{C}$ discharge rate is the highest. Therefore, based on the $0.20 \mathrm{C}$ charge rate, the charge efficiency at different charge rates is obtained. Based on the $0.10 \mathrm{C}$ discharge rate, obtain the discharge efficiency under different discharge rates. Curve fitting is performed on the above experimental results of charging efficiency and discharge rate. Through comparative analysis of the fitting effect of the function relationship under different orders, the fourth degree polynomial is selected as the fitting function of charging efficiency, and the third degree polynomial is selected as the discharge efficiency. The fitting function, fitting curve and fitting error analysis are shown in Figure 5.

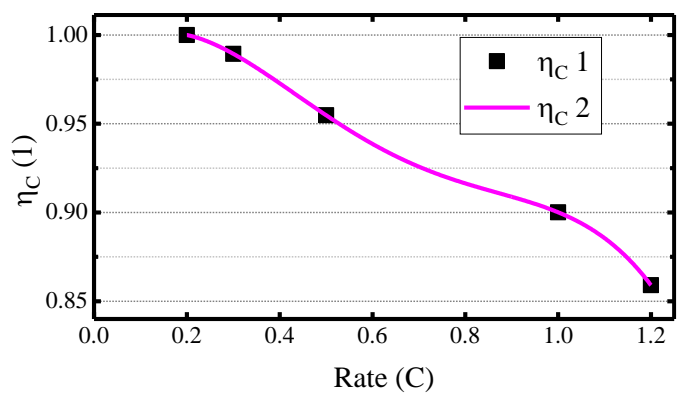

(a) Charging efficiency fitting curve

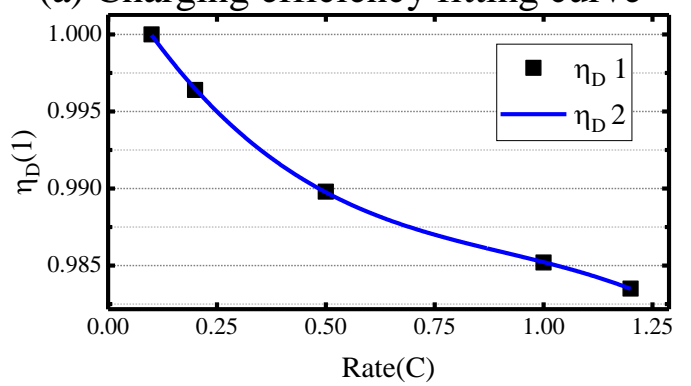

(c) Fitting curve of discharge efficiency

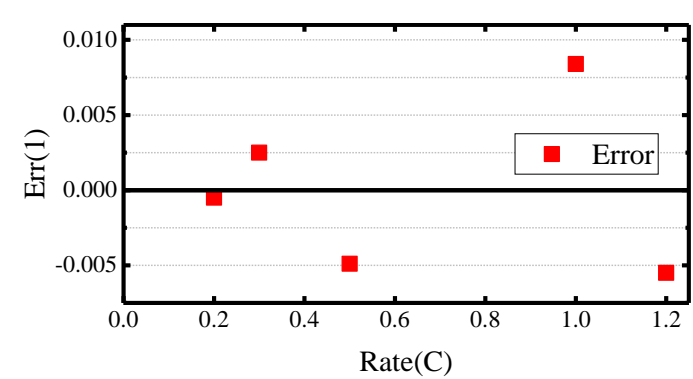

(b) Charge efficiency fitting error

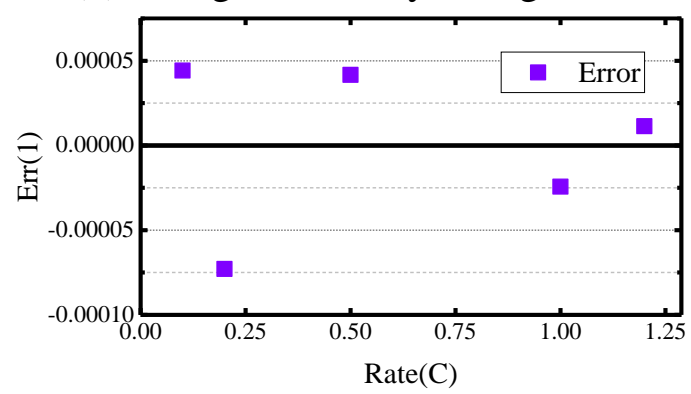

(d) Fitting error of discharge efficiency

Figure 6. Charge and discharge efficiency fitting curve and fitting error analysis chart

In Fig. 6 (a) and (c), $\eta_{C} 1$ and $\eta_{D} 1$ respectively represent the experimental data sets of charging efficiency and discharge efficiency, $\eta_{C} 2$ and $\eta_{D} 2$ respectively represent the function curves of charging efficiency and discharging efficiency. The abscissa represents the charge-discharge current ratio, whose unit is $\mathrm{C}$. The function expression of the fitted curve is shown in Equation (22).

$$
\left\{\begin{array}{l}
f\left(\varepsilon_{C}\right)=-0.6462 * \varepsilon_{C}{ }^{4}+1.6852 * \varepsilon_{C}{ }^{3}-1.4626 * \varepsilon_{C}{ }^{2}+0.3471 * \varepsilon_{C}+0.9766 \\
f\left(\varepsilon_{D}\right)=-0.01609 * \varepsilon_{D}{ }^{3}+0.04399 * \varepsilon_{D}{ }^{2}-0.04690 * \varepsilon_{D}+1.00422
\end{array}\right.
$$

Fig. 6 (b) and (d) are the fitting errors of charging efficiency and discharging efficiency. According to the experimental results, the fitting equation used has a good effect on the simulation of the working characteristics of the lithium-ion battery pack. By embedding the impact of charging and discharging efficiency on the SOC estimation process, the SOC estimation accuracy of the lithium-ion battery pack is improved. 


\subsection{Feasible region analysis of dynamic function}

Through theoretical analysis and experimental research, the feasible region of dynamic function is explored, and the value of constant factor in dynamic function is preliminarily determined, so that the regulation of dynamic function can reach the optimal level. The discussion results of feasible domains of dynamic functions under different constant factor values are shown in Fig. 7.
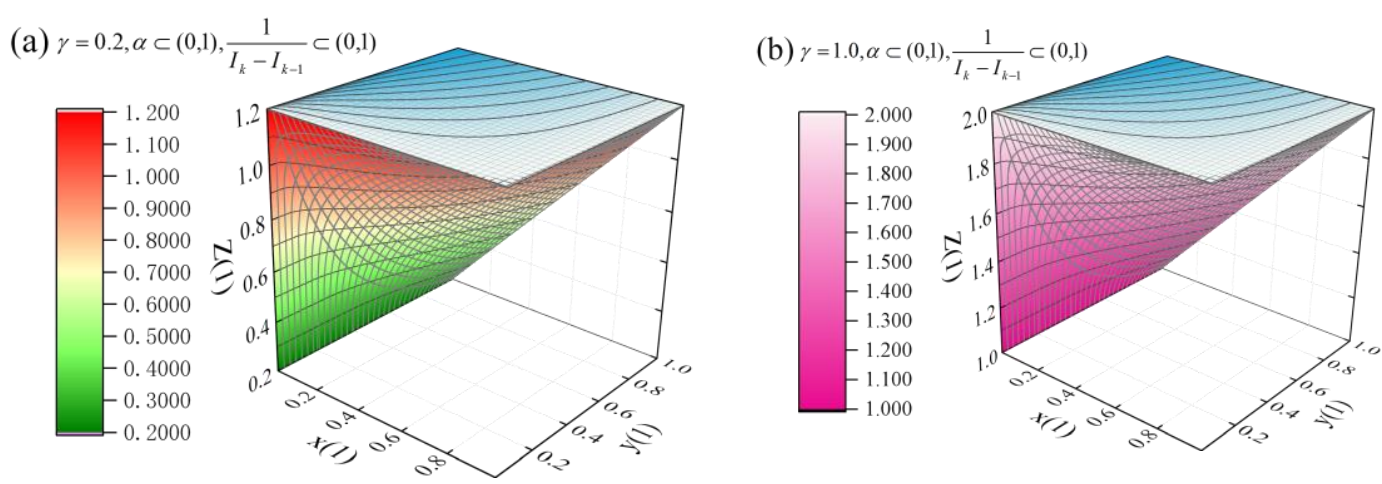

Figure 7. Results of the feasible region of weight constraint factors

In Fig. 7, the z-axis in the three-dimensional graph represents the value range of the dynamic function, and the $\mathrm{x}$-axis and $\mathrm{y}$-axis represent $\frac{1}{\left|I_{k}-I_{k-1}\right|}$ and $\alpha$ respectively. According to theoretical analysis: when $\phi<1$, it will cause the dynamic function "weak adjustment"; when it is $\phi>1$, it will cause the dynamic function to "over-regulate"; when it is 3 , the dynamic function loses its regulating effect. In order to ensure that the adjustment ability of the dynamic function is kept optimal, the value range of the dynamic function is required to be $\phi \subset(1.0,2.0)$. According to the experimental results shown in Fig. 7, only $\gamma=1$ meets the requirements of optimal adjustment strength. Therefore, the subsequent model and algorithm verification research experiments in this paper are all based on $\gamma=1$.

\subsection{Algorithm verification}

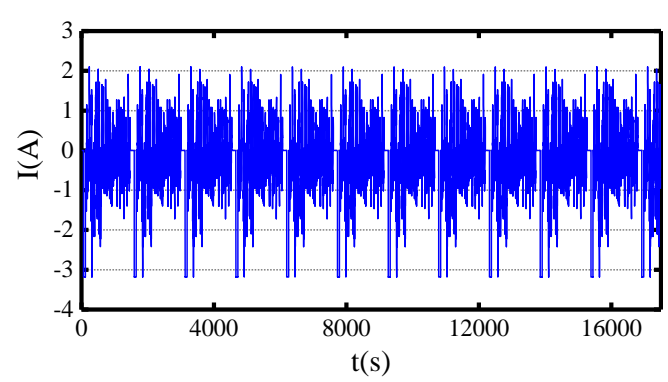

(a) Current curve

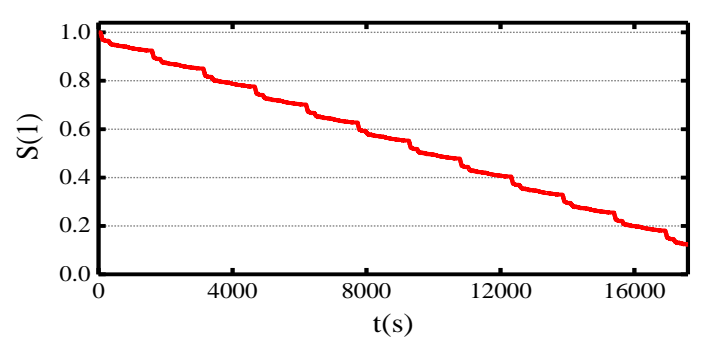

(b) SOC curve 


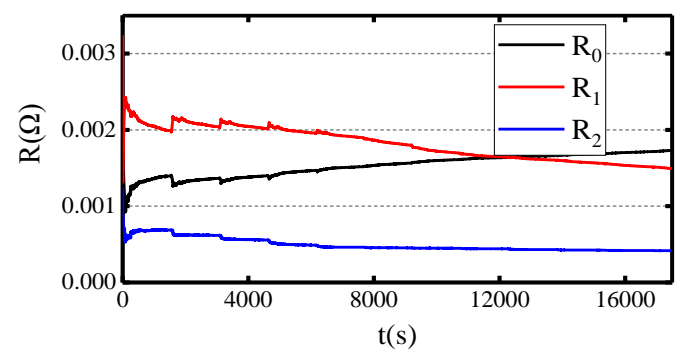

(c) Resistance identification result curve

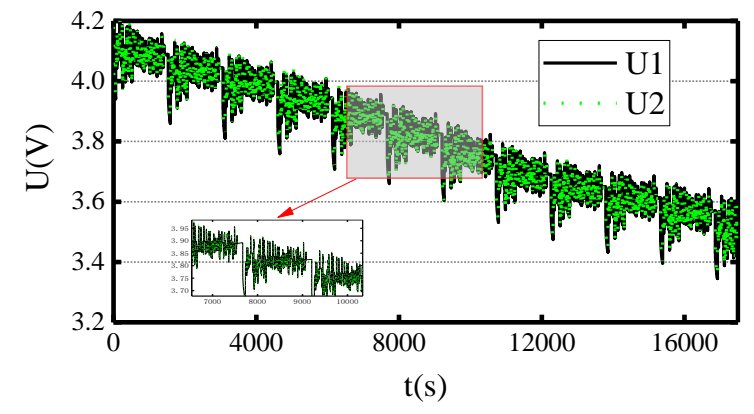

(e) Terminal voltage comparison curve

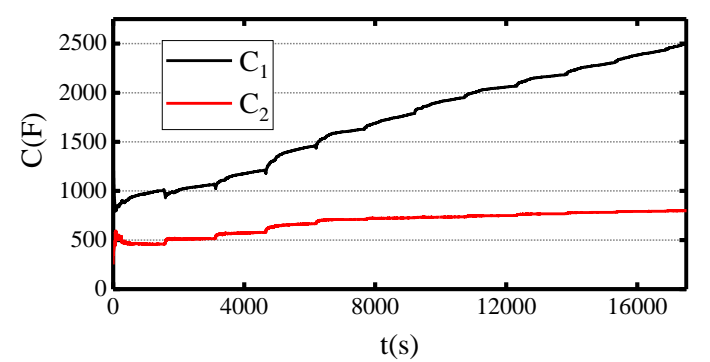

(d) Capacitance identification result curve

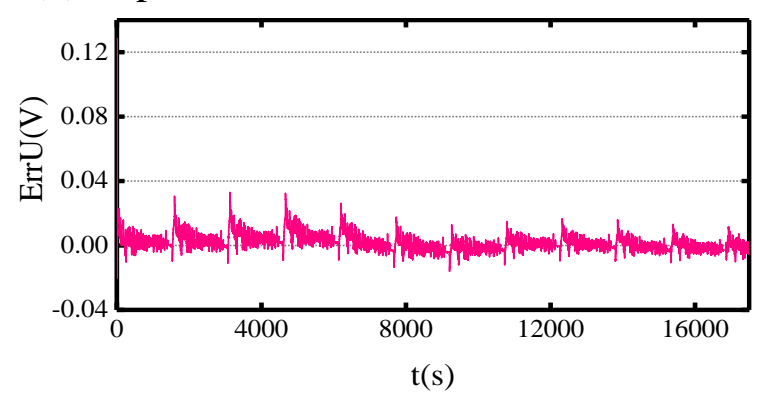

(f) Terminal voltage error curve

Figure 8. Related experimental curves and model error curve under the UDDS working conditions.

MILS algorithm can make full use of new information at every moment and improve data utilization efficiency. In order to verify the accuracy of the E-DCP model mentioned above and the accuracy of MILS, the UDDS working condition is used to conduct online parameter identification of the single cell in the battery pack by using the experimental samples of the selected lithium-ion battery pack and the battery test platform. The experimental process diagram and model error results are shown in Figure 7.

In Fig. 8, U1 represents the actual terminal voltage, $\mathrm{U} 2$ represents the estimated value of the terminal voltage based on the MILS algorithm. $\mathrm{R}_{0}, \mathrm{R}_{1}, \mathrm{R}_{2}, \mathrm{C}_{1}$ and $\mathrm{C}_{2}$ are the parameters to be identified in the E-DCP model. It can be seen from Fig. 8(c) and (d) that in the entire MILS algorithm iteration process, due to the existence of systematic errors, the resistance value and terminal voltage errors in the initial model of identification are relatively large, as the iteration of the MILS algorithm progresses, each parameter identification results tend to be smooth, indicating that the method of online identification of E-DCP model parameters based on the MILS algorithm is more stable. In addition, it can be seen from Fig. 8(f) that due to the existence of system errors, the maximum error of terminal voltage based on MILS algorithm in the initial identification stage is $0.14 \mathrm{~V}$. After the algorithm is stable, the error does not exceed $0.04 \mathrm{~V}$, and its accuracy is $99.05 \%$. The accuracy of E-DCP model combined with online parameter identification algorithm is $1.43 \%$ higher than that in reference [34] (the maximum error is $0.12 \mathrm{~V}$ ) and $3.7 \%$ higher than that in reference [35] (the maximum error is $0.2 \mathrm{~V}$ ), which better verifies the accuracy of the E-DCP model and the accuracy of MILS. Laid the experimental foundation for the follow-up based on SOC estimation.

On the basis of the above theoretical analysis, combined with Lithium-ion battery pack sample, using battery management experiment platform, design the complex charge-discharge cycle test 
condition of experiment, and validate the improved algorithm in the environment of high system input rate stability, the experiment error analysis of the correlation curve and curve as shown in Fig. 9.

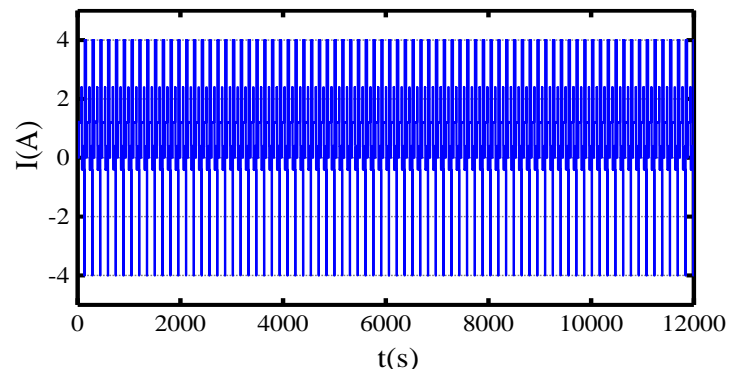

(a) Current curve

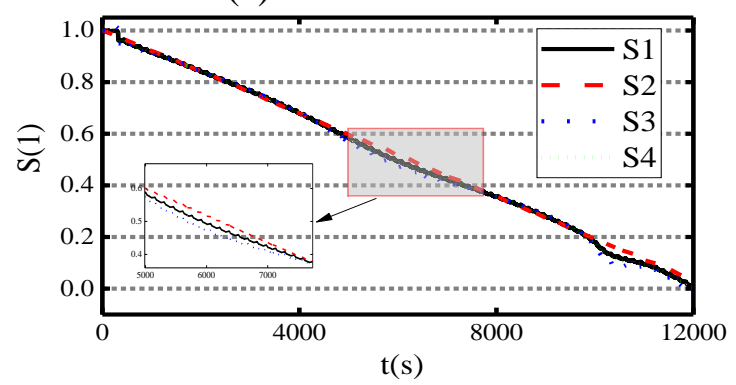

(c) SOC value comparison curve

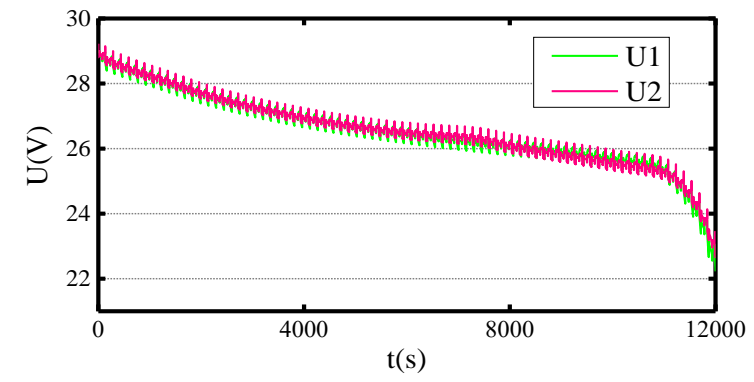

(b) Terminal voltage comparison curve

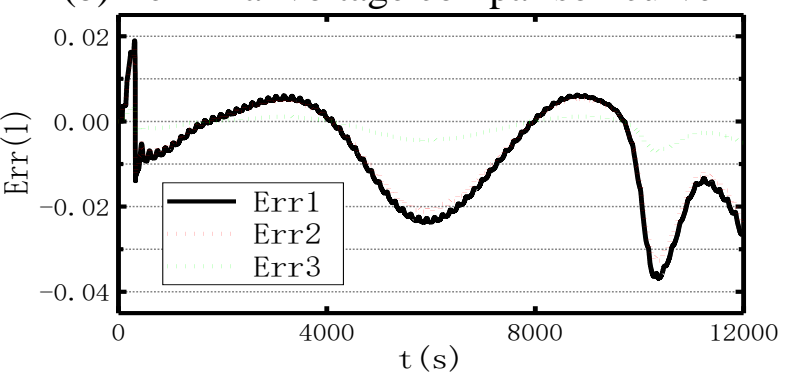

(d) SOC error comparison curve

Figure 9. UDDS test and error comparison

In Fig. 9, U1 represents the actual terminal voltage, and U2 represents the estimated value of the terminal voltage based on the MILS algorithm. S1 represents the true value of the SOC, S2 is the estimated value of the EKF algorithm, S3 is the estimated SOC value of the EKF algorithm based on capacity correction, and S4 is the estimated value of the SOC based on the FC-DEKF algorithm. Err1 is the estimation error of the traditional EKF algorithm, Err2 is the estimation error of the EKF algorithm based on capacity correction, and Err3 is the estimation error based on the FC-DEKF algorithm. It can be seen from Fig. 9(e) that the terminal voltage tracking effect is better, which further verifies the reliability of the online identification of E-DCP model parameters based on the MILS algorithm. In addition, it can be seen from Fig. 9(d) that the estimation effect of the traditional EKF algorithm is not ideal, with a maximum error of $3.8 \%$; the estimation effect of the capacity-corrected EKF algorithm is slightly better than the traditional EKF algorithm, but the error is still large, the maximum error it is $3.1 \%$, and there are large fluctuations in the iteration process; the estimation effect of the FC-EKF algorithm is significantly higher than the previous two algorithms, and its maximum error does not exceed $1 \%$, its estimation accuracy is $2 \%$ higher than that in reference [36] (the estimation accuracy is $3 \%$ ) and $1 \%$ higher than that in reference [37] (the estimation accuracy is $2 \%$ ). The error fluctuation of the algorithm during the entire iteration process is small, indicating under the modified function of FCDEKF algorithm, the BMS system has high robustness. Taken together, the E-DCP model proposed in this paper is of high accuracy, and the FC-DEKF algorithm proposed is superior to the traditional EKF algorithm. 


\section{CONCLUSION}

Through theoretical analysis and experimental research, this paper explores the state estimation method of lithium-ion battery pack. In order to better reflect the dynamic performance of power lithiumion batteries, combined with the advantages of the empirical models of various electrochemical models, a new type of composite E-DCP model is proposed under the conditions of meeting its safe and reliable energy supply requirements. Aiming at the problems of low data utilization and heavy workload of traditional offline parameter identification, the MILS algorithm is used to identify the parameters in the E-DCP model online to improve the efficiency of data utilization and the accuracy of parameter identification. Aiming at the situation that the traditional EKF algorithm is difficult to estimate the error is not ideal when the system input change rate is large, combined with the analysis of the state space description method, the battery charge and discharge efficiency function is introduced to dynamically modify the battery capacity, and the dynamic function is used to correct the Karl in EKF. Mann gain was improved, and a new FC-DEKF algorithm was proposed to achieve accurate estimation of battery pack operating characteristics. UDDS and complex charge-discharge cycle test results show that the maximum error of terminal voltage does not exceed $0.14 \mathrm{~V}$, the accuracy is $96.7 \%$, and the error of MILS algorithm combined with FC-DEKF algorithm for SOC estimation is within 1\%, which is suitable for high-power lithium State estimation of ion battery pack.

NOMENCLATURE

The symbols used in this research can be described as shown in Table 3.

Table 3. List of symbols

\begin{tabular}{|l|l|l|ll|}
\hline Symbol & Description & Symbol & Description \\
\hline E-DCP & $\begin{array}{l}\text { Electrochemistry-Dual Circuit } \\
\text { Polarization }\end{array}$ & UKF & Unscented Kalman Filter & Circuit \\
\hline MILS & Multi-Innovation Least Squares & E-DCP & $\begin{array}{l}\text { Electrochemistry-Dual } \\
\text { Polarization }\end{array}$ \\
\hline EKF & Extended Kalman Filter & RC & resistance-capacitance \\
\hline UDDS & Urban Dynamometer Driving Schedule & BMS & Battery Management System & \\
\hline SOC & State Of Charge & OCV & Open Circuit Voltage \\
\hline
\end{tabular}

\section{ACKNOWLEDGMENTS}

This research was supported by National Natural Science Foundation (No. 61801407). Thanks to the sponsors. CF would like to express his gratitude to RGU for its support.

\section{References}

1. Y. Zhang, X.F. Song, R.T. Huang, Y.W. Ye, and H.R. Li, J. Mater. Sci., 55 (2020) 10740.

2. K.E. Ouardi, M. Dahbi, C. Hakim, M. O. Güler, H. Akbulut, A. E. Bouari, and I. Saadoune, J. Appl. Electrochem., 50 (2020) 583.

3. N.M. Jobst, A. Hoffmann, A. Klein, and A. Klein, ChemSusChem., 13 (2020). 
4. Y.Z. Wang, G.R. H, Y.B. Cao, Z.D. Peng, X.W. Lai, X.M. Xie, and K. Du, Electrochim. Acta, 354 (2020) 136743.

5. M.J. Yu, Y. Li, I. Podlubny, F. Gong, Y. Sun, Q. Zhang, Y.L. Shang, B. Duan, and C.H. Zhang, J. Adv. Res., 25 (2020) 49.

6. G.W. Richardson, J.M. Foster, R. Ranom, C.P. Please, and A.M. Ramos, J. Appl. Math., 2020.

7. M. Mirsalehian, and R. Beykirch, ATZ worldwide, 122 (2020) 36.

8. K.L. Liu, X.S. Hu, Z.B. Wei, Y. Li, and Y. Jang, IEEE Trans. Transp. Electrif., 5 (2020) 1225.

9. $\quad$ X.B. Han, M.G. Ouyang, L.G. Lu, and J.Q. Li, J. Power Sources, 278 (2015) 814.

10. Z.Y. Zhang, L.Z. Zhang, L. Hu, and C.X. Huang, Int. J. Energy Res., 44 (2020).

11. H. Yao, X. Jia, Q. Zhao, Z.J. Chen, and B. Guo, IEEE Access, 99 (2020) 1.

12. S. Potrykus, F. Kutt, J. Nieznański, and F.J.F. Morales, Energies, 13 (2020) 2411.

13. Y.Q Liu, Y.G. Liao, and M.C. Lai, Energies, 13 (3) (2020) 638.

14. D. Clerici, F. Mocera, and A. Somà, Energies, 13 (2020) 1.

15. M.G. Hennessy, and I.R. Moyles, Appl. Math. Modell., 80 (2020) 724.

16. Y. Lin, Y.S. Cai, Y.X. Yang, and Z.W. Deng, Appl. Energy, 257 (2020) 114006.

17. S. Li, K. Li, E. Xiao, R. Xiong, and P. Fischer, J. Power Sources, 445 (2020) 227282.

18. J. Hou, Y. Yang, and T. Gao, Int. J. Energy Res., 44 (5) 2020.

19. X.W. Guo, X.Z. Xu, J.H. Geng, X. Hua, and Z. Liu, Appl. Sci., 9 (2019) 4177.

20. L. Guo, J.Q. Li, and Z.J. Fu, Energy Procedia, 158 (2019) 2599.

21. X. Lai, S.Y. Wang, S.D. Ma, J.Y. Xie, and J.Y. Zhen, Electrochim. Acta, 330 (2019) 135239.

22. C. Bian, H.L. He, S.K. Yang, and T.T. Huang, J. Power Sources, 449 (2019) 227558.

23. Y.J. Wang, and Z.H. Chen, Appl. Energy,260 (2020) 114324.

24. M. Luzi, F.M.F. Mascioli, M. Paschero, and A. Rizzi, IEEE Trans. Neural Networks Learn. Syst., 31 (2) (2020) 371.

25. C.F. Yang, X.Y. Wang, Q.H. Fang, H.F. Dai, and X.Z. Wei, J. Storage Mater., 29 (2020) 101250.

26. C.N. Van, and T.N. Vinh, Appl. Sci., 10 (5) (2020) 1896.

27. W. Saikong, C. Sumpavakup, and T. Kulworawanichpong, Int. J. Intell. Eng. Syst., 12 (2019) 81.

28. M. Luzi, F.M.F. Mascioli, M. Paschero, and A. Rizzi, IEEE Trans. Neural Networks Learn. Syst., 31 (2020) 371.

29. J.Y. Luo, J.K. Peng, and H.W. He, Energy Procedia, 158 (2019) 3421.

30. C.G. Guo, G. Yuan, C.G. Zhu, X.P. Wang, and X. Cao, IOP Conf. Ser.: Mater. Sci. Eng., 486 (2019) 657.

31. J. Hou, Y. Yang, and T. Gao, Int. J. Energy Res., 44 (2020) 3510.

32. Y. Xu, M. Hu, A. Zhou, Y.X. Li, and C.C. Gong, Appl. Math. Modell., 77 (2020) 1255.

33. M.G. Hennessy, and I.R. Moyles, Appl. Math. Modell., 80 (2020) 724.

34. S.G. Yao, L. Peng, M. Xiao, J. Cheng, and L. Xu, Int. J. Electrochem. Sci., 13 (2018) 4455.

35. W. Cao, S.L. Wang, C. Fernandez, C.Y. Zou, C.M. Yu, and X.X. Li, Energy Sci. Eng., 7 (2019) 1544.

36. H.H. Li, X.Y. Wang, A. Saini, Y.Q. Zhu, and Y.P. Wang, Int. J. Electrochem. Sci., 15 (2020) 3807.

37. S.L. Wang, C. Fernandez, M.J. Chen, L. Wang, and J. Su, J. Cleaner Prod., 185 (2018) 187.

(C) 2020 The Authors. Published by ESG (www.electrochemsci.org). This article is an open access article distributed under the terms and conditions of the Creative Commons Attribution license (http://creativecommons.org/licenses/by/4.0/). 